\title{
Elementarisierung von Benkos Lösung des 3. Hilbertschen Problems
}

\author{
Erich Ch. Wittmann \\ Erich Ch. Wittmann studierte Mathematik und Physik für das Höhere Lehramt und \\ promovierte in Gruppentheorie an der Universität Erlangen. Seit 1970 ist er Professor \\ für Elementarmathematik und Didaktik der Mathematik an der Universität Dortmund. \\ Er ist Mitbegründer des Projekts „mathe 2000“.
}

Sind zwei beliebige Tetraeder mit gleichen Grundflächen und gleichen Höhen stets zerlegungsgleich oder lassen sie sich mit kongruenten Polyedern zu zerlegungsgleichen Körpern ergänzen?

So formulierte D. Hilbert bei dem zweiten internationalen Mathematikerkongress in Paris das dritte seiner 23 Probleme. Kaum ein Jahr später wurde dieses Problem bereits gelöst: M. Dehn bewies, dass der Würfel und das volumengleiche reguläre Tetraeder nicht zerlegungsgleich sind ([4]). Bei Versuchen den Dehnschen Beweis zu vereinfachen, gelang erst V.G. Boltianskii eine markante Verbesserung ([3]). Vor einigen Jahren hat D. Benko die Fachwelt mit einer unerwartet einfachen Lösung des 3. Hilbertschen Problems überrascht ([2]). Entscheidendes Hilfsmittel für seinen Beweis ist eine bestimmte Approximation reeller Zahlen durch rationale Zahlen (Lemma 1 bei Benko). M. Aigner und G. Ziegler haben den Ansatz von Benko aufgegriffen und in der dritten Auflage ihres berühmten Buchs der

Zwei Polygone, die sich in Polygone zerlegen lassen, die paarweise kongruent sind, haben wegen der Additivität des Flächeninhalts offensichtlich den gleichen Inhalt. Dass umgekehrt inhaltsgleiche Polygone auch zerlegungsgleich sind, lässt sich mit elementaren Mitteln beweisen (W. Bolyai 1832, P. Gerwien 1833). Ob im Raum aus der Volumengleichheit von Polyedern ebenfalls die Zerlegungsgleichheit folgt, war lange offen und wurde im Jahr 1900 von D. Hilbert als 3. Problem in seine berühmte Liste von 23 Problemen aufgenommen. M. Dehn hat bereits ein Jahr später mit Mitteln der Höheren Mathematik bewiesen, dass die Begriffe volumengleich und zerlegungsgleich bei Polyedern nicht äquivalent sind. Im vorliegenden Beitrag wird im Anschluss an Benko 2007 ein Beweis geliefert, der mit elementaren Mitteln auskommt. Entscheidend ist dabei eine bestimmte Approximation reeller durch rationale Zahlen. 
Beweise eine eigene Version veröffentlicht. Darin spielt das Kegellemma eine zentrale Rolle. $\mathrm{Zu}$ dessen Beweis setzen sie allerdings stärkere Mittel ein, die aber natürlich viel weiter reichen ([1, S. 61-70]).

Ziel der vorliegenden Note ist eine weitere Elementarisierung und Vereinfachung des Beweises der Nichtäquivalenz von Zerlegungsgleichheit und Volumengleichheit bei Polyedern unter Beschränkung auf Mittel der Schulmathematik. Dabei spielt die Darstellung reeller Zahlen durch unendliche Dezimalbrüche die entscheidende Rolle.

Das folgende Lemma ist eine Variante von Lemma 1 in [2]:

Lemma. Gegeben sei eine Menge $\left\{a_{1}, \ldots, a_{n}\right\}$ positiver reeller Zahlen und $m$ eine beliebige natürliche Zahl. Dann existieren nicht negative rationale Zahlen $\frac{p_{i}}{q}, i=1, \ldots, n$, sodass für $i=1, \ldots, n$ gilt $\left|a_{i}-\frac{p_{i}}{q}\right| \leq \frac{1}{10^{m} \cdot q}$.

Beweis. Da bei der Dezimalbruchdarstellung positiver reeller Zahlen die ersten $m$ Stellen nach dem Komma auf $10^{m}$ verschiedene Arten mit den Ziffern 0 bis 9 belegt werden können, gibt es für beliebiges $m$ in einer unendlichen Menge positiver reeller Zahlen unendlich viele, deren Dezimalbruchentwicklungen in den ersten $m$ Stellen nach dem Komma übereinstimmen (Dirichletsches Schubfachprinzip). Die Differenz zweier solcher Zahlen unterscheidet sich von einer natürlichen Zahl um höchstens $10^{-m}$.

Die Menge der Vielfachen $t \cdot a_{1}, t \in \mathbb{N}$, ist eine unendliche Menge. Daher existiert eine unendliche Teilmenge $\mathbb{N}_{1}$ von $\mathbb{N}$, sodass die Vielfachen $t \cdot a_{1}, t \in \mathbb{N}_{1}$, in den ersten $m$ Stellen nach dem Komma übereinstimmen. Die Menge $t \cdot a_{2}, t \in \mathbb{N}_{1}$, ist ebenfalls unendlich. Daher gibt es eine unendliche Teilmenge $\mathbb{N}_{2}$ von $\mathbb{N}_{1}$, sodass die Vielfachen $t \cdot a_{2}, t \in \mathbb{N}_{2}$, ebenfalls in den ersten $m$ Stellen nach dem Komma übereinstimmen. Durch wiederholte Anwendung dieser Konstruktion gelangt man nach $n$ Schritten zu einer unendlichen Teilmenge $\mathbb{N}_{n}$ von $\mathbb{N}$ mit der Eigenschaft, dass für jedes $i=1, \ldots, n$ die Zahlen $t \cdot a_{i}, t \in \mathbb{N}_{n}$, jeweils in den ersten $m$ Stellen nach dem Komma übereinstimmen.

Für zwei beliebige Zahlen $r, s \in \mathbb{N}_{n}$ mit $r<s$ folgt, wenn $q:=s-r$ gesetzt wird, dass sich $q \cdot a_{i}$ für $i=1, \ldots, n$ um höchstens $10^{-m}$ von einer ganzen Zahl $p_{i} \geq 0$ unterscheidet und daher $a_{i}$ von der rationalen Zahl $\frac{p_{i}}{q}$ um höchstens $\frac{10^{-m}}{q}=\frac{1}{10^{m} \cdot q}$.

Zusatz 1. Wenn $s$ hinreichend groß gewählt wird, kann man erreichen, dass alle $p_{i}$ positiv sind, was für die folgenden Überlegungen aber irrelevant ist.

Zusatz 2. Statt von der unendlichen Menge $\mathbb{N}$ kann man auch von einer endlichen Menge $\mathbf{M}$ natürlicher Zahlen ausgehen, die mindestens $\left(10^{m}\right)^{n}+1$ Elemente enthält. Nach dem Schubfachprinzip ergibt sich nach $n$ Schritten eine Menge $\mathbf{M}_{n}$ mit mindestens zwei Elementen, welche in Bezug auf $a_{1}, \ldots, a_{n}$ die gleichen Eigenschaften wie $\mathbb{N}_{n}$ aufweist.

Zusatz 3. Aus dem Lemma lässt sich mühelos das Kegellemma von Aigner/Ziegler ([1, S. 64]) herleiten. Man braucht $m$ nur hinreichend groß zu wählen und kann dann eine ähnliche Abschätzung vornehmen wie unten beim Beweis von Satz 1.

Der Beweis des Theorems 2 von Benko, welches die Lösung des Hilbertschen Problems liefert, lässt sich mit Hilfe des folgenden Begriffs, der eine anschaulichere Fassung des Dehnschen Funktionals ist, übersichtlicher führen. 
Definition. Für ein Polyeder $P$ wird unter der gewichteten Winkelsumme $S(P)$ des Polyeders die Summe $\sum l\left(e_{j}\right) \cdot \alpha_{j}$ verstanden. Summiert wird dabei über alle Kanten $e_{j}$ des Polyeders. $l\left(e_{j}\right)$ ist die Länge von $e_{j}$ und $\alpha_{j}$ der zu der Kante $e_{j}$ gehörende Diederwinkel. Die gewichtete Winkelsumme $S\left(P_{1}, \ldots, P_{k}\right)$ einer Menge $\left\{P_{1}, \ldots, P_{k}\right\}$ von Polyedern wird definiert als $S\left(P_{1}\right)+\cdots+S\left(P_{k}\right)$.

Die Zerlegung eines Polyeders $P$ in Polyeder $P_{1}, \ldots, P_{k}$ gibt Anlass, die einzelnen Summanden von $S\left(P_{1}, \ldots, P_{k}\right)$ in folgender kanonischer Weise zu zerlegen: Bei der Zerlegung von $P$ wird jede Kante $e$ eines Polyeders $P_{i}$ durch die Ecken der anderen Polyeder, die auf ihr liegen, sowie durch ihre Schnittpunkte mit Kanten der anderen Polyeder in Teilstrecken zerlegt.

Der Beitrag jeder Kante $e$ zur Summe $S\left(P_{1}, \ldots, P_{k}\right)$ der Diederwinkel kann daher auch dadurch ausgedrückt werden, dass man die Länge jeder Teilstrecke von $e$ mit dem zugehörigen Diederwinkel multipliziert und die Produkte aufsummiert. Diese Darstellung heißt die durch $P$ verfeinerte Darstellung $S_{P}\left(P_{1}, \ldots, P_{k}\right)$ der gewichteten Winkelsumme $S\left(P_{1}, \ldots, P_{k}\right)$.

Wenn sich ein zweites Polyeder $Q$ in die gleichen Polyeder $P_{1}, \ldots, P_{k}$ zerlegen lässt, in diesem Fall sind $P$ und $Q$ zerlegungsgleich und volumengleich, ergibt sich eine analoge Aufspaltung der Kanten von $P_{1}, \ldots, P_{k}$ und die durch $Q$ verfeinerte Darstellung $S_{Q}\left(P_{1}, \ldots, P_{k}\right)$ der Winkelsumme $S\left(P_{1}, \ldots, P_{k}\right)$.

Offensichtlich gilt $S_{P}\left(P_{1}, \ldots, P_{k}\right)=S\left(P_{1}, \ldots, P_{k}\right)=S_{Q}\left(P_{1}, \ldots, P_{k}\right)$.

Sei nun $\left\{L_{1}, \ldots, L_{n}\right\}$ die Menge sämtlicher Kanten der Polyeder $P_{1}, \ldots, P_{k}$ und sämtlicher Teilstrecken, die aus den Zerlegungen von $P$ und $Q$ in $P_{1}, \ldots, P_{k}$ resultieren. Die natürliche Zahl $m$ wird so groß gewählt, dass $N<10^{m}$ gilt. Aus dem obigen Lemma folgt, dass sich die reellen Längen $l\left(L_{1}\right), \ldots, l\left(L_{N}\right)$ durch nicht negative rationale Zahlen $\frac{p_{1}}{q}, \ldots, \frac{p_{N}}{q}$ so gut approximieren lassen, dass der Fehler jeweils höchstens $\frac{1}{10^{m} \cdot q}$ ist.

Satz 1. Die approximativen gewichteten Winkelsummen $S^{*}\left(P_{1}, \ldots, P_{k}\right), S_{P}^{*}\left(P_{1}, \ldots, P_{k}\right)$ und $S_{Q}^{*}\left(P_{1}, \ldots, P_{k}\right)$, die entstehen, wenn in den gewichteten Winkelsummen $S\left(P_{1}, \ldots\right.$, $\left.P_{k}\right), S_{P}\left(P_{1}, \ldots, P_{k}\right)$ und $S_{Q}\left(P_{1}, \ldots, P_{k}\right)$ die Längen $l\left(L_{i}\right)$ für $i=1, \ldots, N$ durch ihre rationalen Näherungen $\frac{p_{i}}{q}$ ersetzt werden, sind gleich.

Beweis. Für jede Kante $e$ eines Polyeders $P_{i}$ ist die Summe der Längen der bei der Zerlegung von $P$ induzierten Teilstrecken, gleich der Länge von $e$, ebenso die Summe der Längen der bei der Zerlegung von $Q$ induzierten Teilstrecken. Der approximative Wert von $e$ und die Summe der approximativen Längen der Teilstrecken von $e$ sind jeweils Brüche mit dem Nenner $q$, die sich um höchstens $\frac{N}{10^{m} \cdot q}<\frac{1}{q}$ unterscheiden. Die Zähler und daher auch die Brüche müssen somit gleich sein. Bei dieser Abschätzung wird benutzt, dass die Anzahl der Teilstrecken jeder Kante $e$ jeweils kleiner als $N$ ist.

Satz 2. Für zerlegungsgleiche Polyeder $P$ und $Q$ ist die Differenz $S^{*}(P)-S^{*}(Q)$ ein rationales Vielfaches von $\pi$.

Beweis. Wir fassen in den Summen $S_{P}^{*}\left(P_{1}, \ldots, P_{k}\right)$ und $S_{Q}^{*}\left(P_{1}, \ldots, P_{k}\right)$ diejenigen Terme zusammen, die zur gleichen Teilstrecke $L_{i}$ gehören. Dabei sind drei Fälle zu unterscheiden. 
(1) Wenn $L_{i}$ im Innern von $P$ bzw. $Q$, aber nicht auf einer Seitenfläche eines der Polyeder $P_{1}, \ldots, P_{k}, P$ bzw. $Q$ liegt, summieren sich die zu $L_{i}$ gehörenden Diederwinkel zu $2 \pi$ (Abb. 1). Daher liefert jede solche Teilstrecke den Beitrag $\frac{p_{i}}{q} \cdot 2 \pi$.

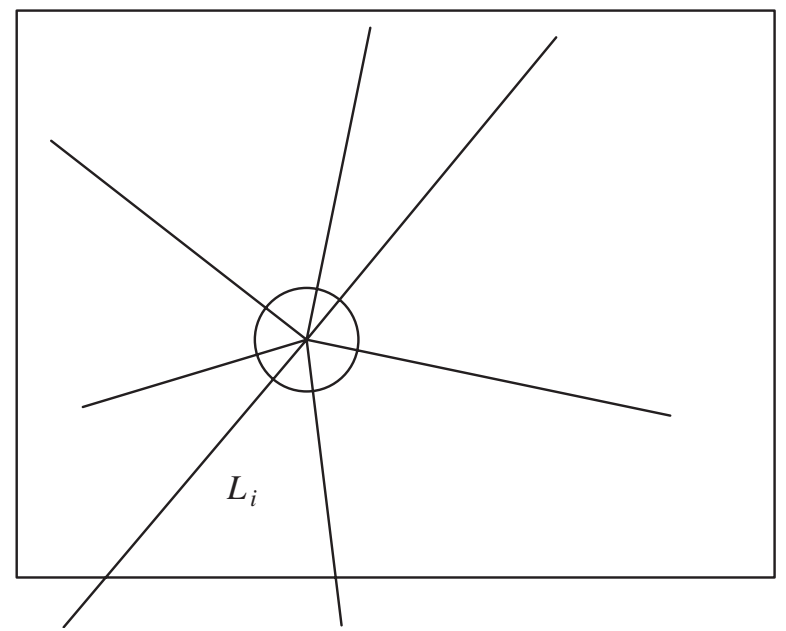

Abb. 1 Orthogonaler Schnitt durch eine Teilstrecke $L_{i}$ entsprechend Fall (1)

(2) Wenn $L_{i}$ im Innern von $P$ bzw. $Q$ und auf einer Seitenfläche eines der Polyeder $P_{1}, \ldots, P_{k}$ oder $P$ bzw. $Q$ liegt, summieren sich die zugehörigen Diederwinkel zu $\pi$ (Abb. 2). Entsprechend ist der Beitrag einer solchen Teilstrecke $\frac{p_{i}}{q} \cdot \pi$.

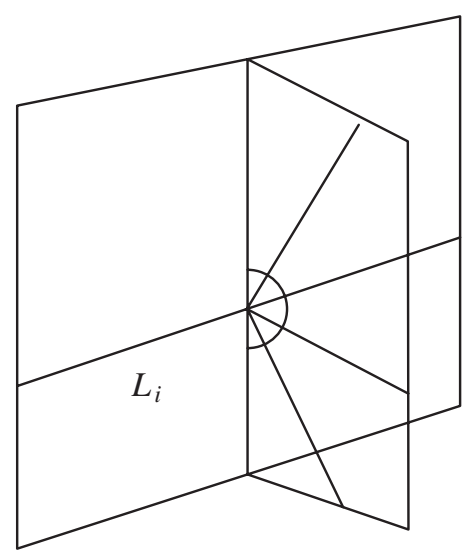

Abb. 2 Orthogonaler Schnitt durch eine Teilstrecke $L_{i}$ entsprechend Fall (2)

(3) Wenn $L_{i}$ auf einer Kante von $P$ bzw. $Q$ liegt, summieren sich die zu $L_{i}$ gehörenden Diederwinkel zum Diederwinkel $\alpha_{j}$ dieser Kante. Die Teilstrecken dieses Typs liefern zu $S_{P}^{*}\left(P_{1}, \ldots, P_{k}\right)$ den Beitrag $S^{*}(P)$ und zu $S_{Q}^{*}\left(P_{1}, \ldots, P_{k}\right)$ den Beitrag 
$S^{*}(Q)$. Somit haben wir

$$
\begin{aligned}
& S_{P}^{*}\left(P_{1}, \ldots, P_{k}\right)=S^{*}(P)+\frac{u}{q} \cdot \pi, \\
& S_{Q}^{*}\left(P_{1}, \ldots, P_{k}\right)=S^{*}(Q)+\frac{v}{q} \cdot \pi
\end{aligned}
$$

mit ganzen Zahlen $u, v$. Aus Satz 1 ergibt $\operatorname{sich} S^{*}(P)-S^{*}(Q)=\frac{u-v}{q} \cdot \pi$.

Anmerkung: Wenn man alle Terme in der letzten Gleichung mit dem gemeinsamen Nenner $q$ multipliziert, folgt für zerlegungsgleiche Polyeder $P$ und $Q$, dass es eine Summe von ganzzahligen Vielfachen der Diederwinkel von $P$ und eine Summe von Vielfachen der Diederwinkel von $Q$ gibt, die sich nur um ein Vielfaches von $\pi$ unterscheiden (Bricardsche Bedingung).

Satz 3. Ein reguläres Tetraeder $T$ und der zu $T$ volumengleiche Würfel $W$ sind nicht zerlegungsgleich.

Beweis (indirekt): Angenommen $T$ und $W$ wären zerlegungsgleich. Dann gäbe es Polyeder $P_{1}, \ldots, P_{k}$, aus denen sich sowohl $T$ als auch $W$ zusammensetzen ließen. Beim Würfel ist der Diederwinkel aller Kanten $\frac{\pi}{2}$, beim Tetraeder gilt für den Diederwinkel $\alpha$ aller Kanten $\cos \alpha=\frac{1}{3}$, wie aus Abb. 3 abzulesen ist. $A M$ und $D M$ sind Höhen und Seitenhalbierende der regulären Dreiecke $A B C$ und $D B C$ und damit gleich lang. Der Fußpunkt $F$ der Höhe $D F$ des Tetraeders ist der Mittelpunkt von $A B C$ und teilt als Schnittpunkt der Seitenhalbierenden die Strecke $A M$ im Verhältnis $2: 1$.

$S^{*}(T)$ ist ein rationales Vielfaches von $\alpha, S^{*}(W)$ ein rationales Vielfaches von $\pi$. Aus Satz 2 folgt, dass es natürliche Zahlen $n$ und $k$ gibt, für die $n \cdot \alpha=k \cdot \pi$ gilt, was $n>1$ einschließt. Aus dieser Winkelbeziehung folgt $\cos n \cdot \alpha=\cos k \cdot \pi= \pm 1$.

Mit Hilfe der Additionstheoreme für Sinus und Kosinus zeigt man durch vollständige Induktion, dass für beliebige $\varphi$ die Beziehung $\cos n \cdot \varphi=T_{n}(\cos \varphi)$ gilt, wobei $T_{n}$ ein Polynom $n$-ten Grades mit ganzzahligen Koeffizienten und $2^{n-1}$ der Koeffizient der höchsten

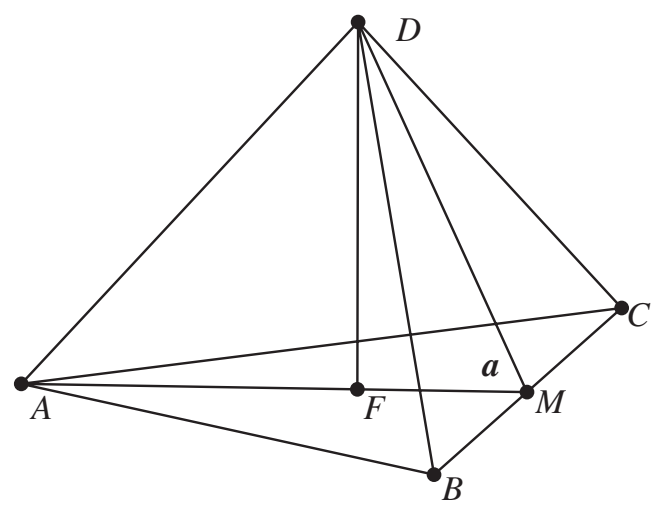

Abb. 3 
Potenz von $T_{n}$ ist. Wegen $\cos \alpha=\frac{1}{3}$ müsste daher eine Gleichung der Form

$$
2^{n-1} \cdot\left(\frac{1}{3}\right)^{n}+a_{n-1} \cdot\left(\frac{1}{3}\right)^{n-1}+\cdots+a_{1} \cdot \frac{1}{3}+a_{0}= \pm 1
$$

mit ganzen Zahlen $a_{i}$ bestehen. Wenn beide Seiten dieser Gleichung mit $3^{n}$ multipliziert werden, erhält man

$$
2^{n-1}+a_{n-1} \cdot 3+\cdots+a_{1} \cdot 3^{n-1}+a_{0} \cdot 3^{n}= \pm 3^{n},
$$

woraus

$$
2^{n-1}= \pm 3^{n}-\left(a_{n-1} \cdot 3+\cdots+a_{1} \cdot 3^{n-1}+a_{0} \cdot 3^{n}\right)
$$

folgt. Die rechte Seite dieser Gleichung ist durch 3, die linke durch 2 teilbar, was ein Widerspruch zur Teilerfremdheit von 2 und 3 ist. Dieser Widerspruch zeigt, dass $T$ und $W$ nicht zerlegungsgleich sein können.

\section{Literatur}

[1] Aigner, M.; Ziegler, G.: Das Buch der Beweise. Springer, Berlin 2009.

[2] Benko, D.: A New Approach to Hilbert's Third Problem. Amer. Math. Monthly 114 (2007), 665-676.

[3] Boltianskii, V.G.: Hilbert's Third Problem. Washington DC: V.H. Winston \& Sons, Washington D.C. 1978.

[4] Dehn, M.: Über raumgleiche Polyeder. Nachr. Akad. Wiss. Göttingen Math.-Phys. Kl. II (1900), 345-354.

Erich Ch. Wittmann

Technische Universität Dortmund

Fakultät für Mathematik

44221 Dortmund, Deutschland

e-mail:wittmann@math.tu-dortmund.de 